\title{
Variable Interhemispheric Asymmetry in Layer V of the Supplementary Motor Area following Cervical Hemisection in Adult Macaque Monkeys
}

\author{
고. Contestabile, ${ }^{1,2}$ R. Colangiulo, ${ }^{2}$ M. Lucchini, ${ }^{1,2}$ E.M. Rouiller, ${ }^{2}$ and ${ }^{\circ}$ E. Schmidlin ${ }^{2}$
}

https://doi.org/10.1523/ENEURO.0280-20.2020

${ }^{1}$ Department of Basic Neuroscience, University of Geneva, Genève CH-1205, Switzerland and ${ }^{2}$ Department of Neurosciences and Movement Sciences, Section of Medicine, Faculty of Sciences and Medicine, Fribourg Center of Cognition, University of Fribourg, Fribourg $\mathrm{CH}-1700$, Switzerland

\section{Visual Abstract}

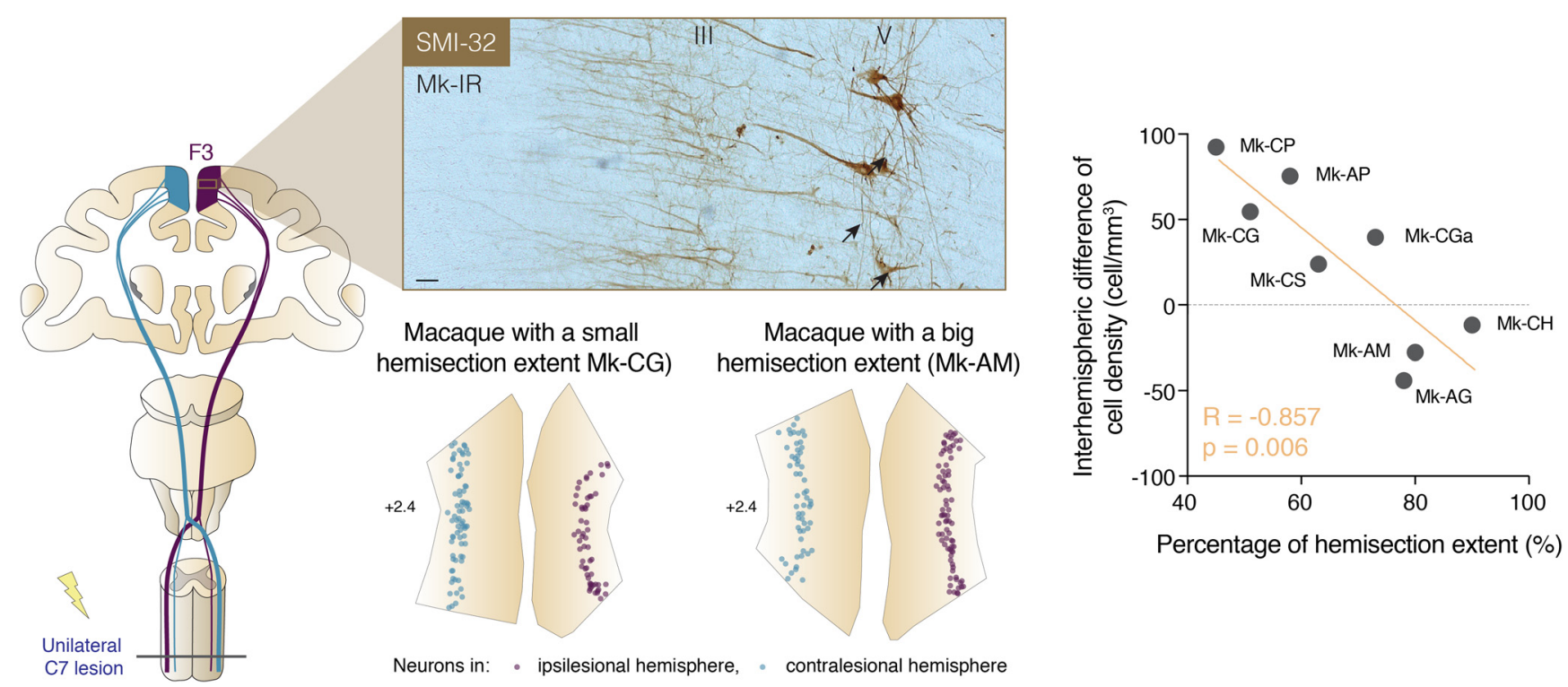

Motor cortical areas from both hemispheres play a role during functional recovery after a unilateral spinal cord injury (SCl). However, little is known about the morphologic and phenotypical differences that a SCl could

\section{Significance Statement}

This study consists in a precise quantification on two different levels of the histological consequences on the long term of a traumatic and sudden unilateral interruption of the corticospinal (CS) tract at cervical level in eight non-human primates (adult macaque monkeys). The lesion affected the density and the morphology of Layer V pyramidal neurons in the supplementary motor area (SMA), in the form of an interhemispheric adaptive asymmetry, correlated to the lesion size and duration of functional recovery. These changes are reminiscent of those observed in SMA after unilateral lesion of the primary motor cortex (M1), suggesting to some extent comparable mechanism of functional motor recovery from unilateral cortical or spinal lesion. The dendritic arborization in the basal dendrites of the SMI-32-positive neurons in Layer V showed a more prominent interhemispheric effect of the lesion than the apical dendrites. 
trigger in corticospinal (CS) neurons of the ipsilesional and contralesional hemisphere. Using an SMI-32 antibody which specifically labeled pyramidal neurons in cortical Layers $\mathrm{V}$, we investigated the impact of a unilateral cervical cord lesion on the rostral part (F6) and caudal part (F3) of the supplementary motor area (SMA) in both hemispheres of eight adult macaque monkeys compared with four intact control monkeys. We observed in F3 (but not in F6) interindividual variable and adaptive interhemispheric asymmetries of SMI-32-positive Layer $\mathrm{V}$ neuronal density and dendritic arborization, which are strongly correlated with the extent of the $\mathrm{SCl}$ as well as the duration of functional recovery, but not with the extent (percentage) of functional recovery.

Key words: corticospinal projections; interhemispheric asymmetry; non-human primate; pyramidal neurons; spinal cord injury; supplementary motor area

\section{Introduction}

In non-human primates, the hand area of the primary motor cortex (M1 or $\mathrm{F} 1$ ) is subdivided in an old $\mathrm{M} 1$ and a new M1 (Rathelot and Strick, 2009). The new M1 is at the origin of the corticomotoneuronal (CM) projection, representing the anatomic support of manual dexterity, a prerogative of primates (Lawrence and Kuypers, 1968; Courtine et al., 2007; Lemon, 2008; Yoshida and Isa, 2018). Although $M 1$ is the main contributor to the corticospinal (CS) projection (including the CM projection), nonprimary motor areas such as the premotor cortex (PM), the SMA (SMA-proper or F3) and the cingulate motor areas (CMA) are also at the origin of CS projections (Luppino et al., 1994; Rouiller et al., 1994, 1996; Dum and Strick, 1996). In particular, SMA projects to the cervical spinal cord, where the motoneurons controlling hand (fingers) motor function are located (Jenny and Inukai, 1983). There is evidence that part of the CS projection from SMA may be CM (Rouiller et al., 1996), but the influence of SMA on hand motoneurons is functionally less strong than the one of M1 (Maier et al., 2002; Boudrias et al., 2010). The multiple representations of the hand in several motor cortical areas (M1, PM, SMA, CMA) of primates is the basis for a vicarious scenario of functional redistribution of hand function control in case of selective and focal lesion affecting a motor structure. For instance, after

Received June 29, 2020; accepted September 3, 2020; First published September 11, 2020.

The authors declare no competing financial interests.

Author contributions: E.S. designed research; A.C. and E.S. performed research; A.C., R.C., M.L., E.M.R., and E.S. analyzed data; A.C., E.M.R., and E.S. wrote the paper.

This work was supported by Swiss National Science Foundation Grants 3161857.00, 310000-110005, 31003A-132465, and 310030B-149643 (to E.M.R.) and 320030-160229 (to E.S.); the National Centre of Competence in Research on "Neural Plasticity and Repair"; the Novartis Foundation; The Christopher Reeves Foundation (Springfield, NJ); and The Swiss Primate Competence Centre for Research.

Acknowledgements: We thank the excellent technical assistance of Véronique Moret, Christine Roulin, and Christiane Marti (histology); Laurent Bossy, Joseph Corpataux, and Jacques Maillard (animal care taking); André Gaillard (mechanics); Bernard Aebischer and Andrea Francovich (electronics); and Laurent Monney (informatics).

Correspondence should be addressed to E. Schmidlin at eric. schmidlin@unifr.ch.

https://doi.org/10.1523/ENEURO.0280-20.2020

Copyright (C) 2020 Contestabile et al.

This is an open-access article distributed under the terms of the Creative Commons Attribution 4.0 International license, which permits unrestricted use, distribution and reproduction in any medium provided that the original work is properly attributed. unilateral lesion of the hand area in M1 functional recovery, although often incomplete, depends on plasticity of intact non-primary motor areas, such as PM (Liu and Rouiller, 1999; Dancause et al., 2005; Hoogewoud et al., 2013; Orczykowski et al., 2018) and/or SMA (McNeal et al., 2010; Morecraft et al., 2015). Although such rearrangement of the cortical motor circuits is believed to occur mostly in the ipsilesional hemisphere, there is still controversy about the role played by the contralesional hemisphere (for the non-human primate, see Morecraft et al., 2016; Savidan et al., 2017). In a recent article from this laboratory, we reported that a unilateral lesion of the M1 hand area led to a variable interhemispheric asymmetry in the detection of Layer V pyramidal neurons in SMA, identified with the marker SMI-32 (Contestabile et al., 2018). This anatomic variable interhemispheric imbalance possibly reflects an adaptive interhemispheric contribution of the bilateral SMA to recovery, depending on the lesion size as well as on the duration of functional recovery (Contestabile et al., 2018). It was argued that these observations may represent a putative anatomic support of diaschisis, originally defined as a "loss of function and electrical activity in an area of the brain because of a lesion in a remote area that is neuronally connected with it" (Finger et al., 2004; see also von Monakow, 1914). In other words, because of a unilateral M1 lesion, the function of the SMA is affected at distance differently on the ipsilesional versus contralesional hemispheres, because the reciprocal connections between M1 and SMA are stronger ipsilaterally than contralaterally, in the intact state. More recently, the concept of diaschisis has been extended to a structural dimension, in the form of "connectional diaschisis," focused on postlesion changes of connectivity in networks/circuits directly or indirectly related to the focal lesion site (Carrera and Tononi, 2014).

In the current study, the main goal was to transpose this concept of interhemispheric adaptable SMA reorganization related to functional recovery from a lesion of $M 1$ (Contestabile et al., 2018) to a cervical spinal cord hemisection. Does a unilateral spinal cord injury (SCl) also affect differently the contralesional versus the ipsilesional SMA, because the CS projection from SMA is predominantly crossed (Fig. 1A,B)? Following a hemisection of the cervical cord in macaque monkeys, it was observed that the CS axotomy did not lead to a retrograde death of CS neurons in M1 (Wannier et al., 2005) but, instead, to a somatic shrinkage of CS neurons in Layer $\mathrm{V}$ of the contralesional M1 (Beaud et al., 2008). As far as the functional recovery from CS tract injury or cervical cord lesion is 

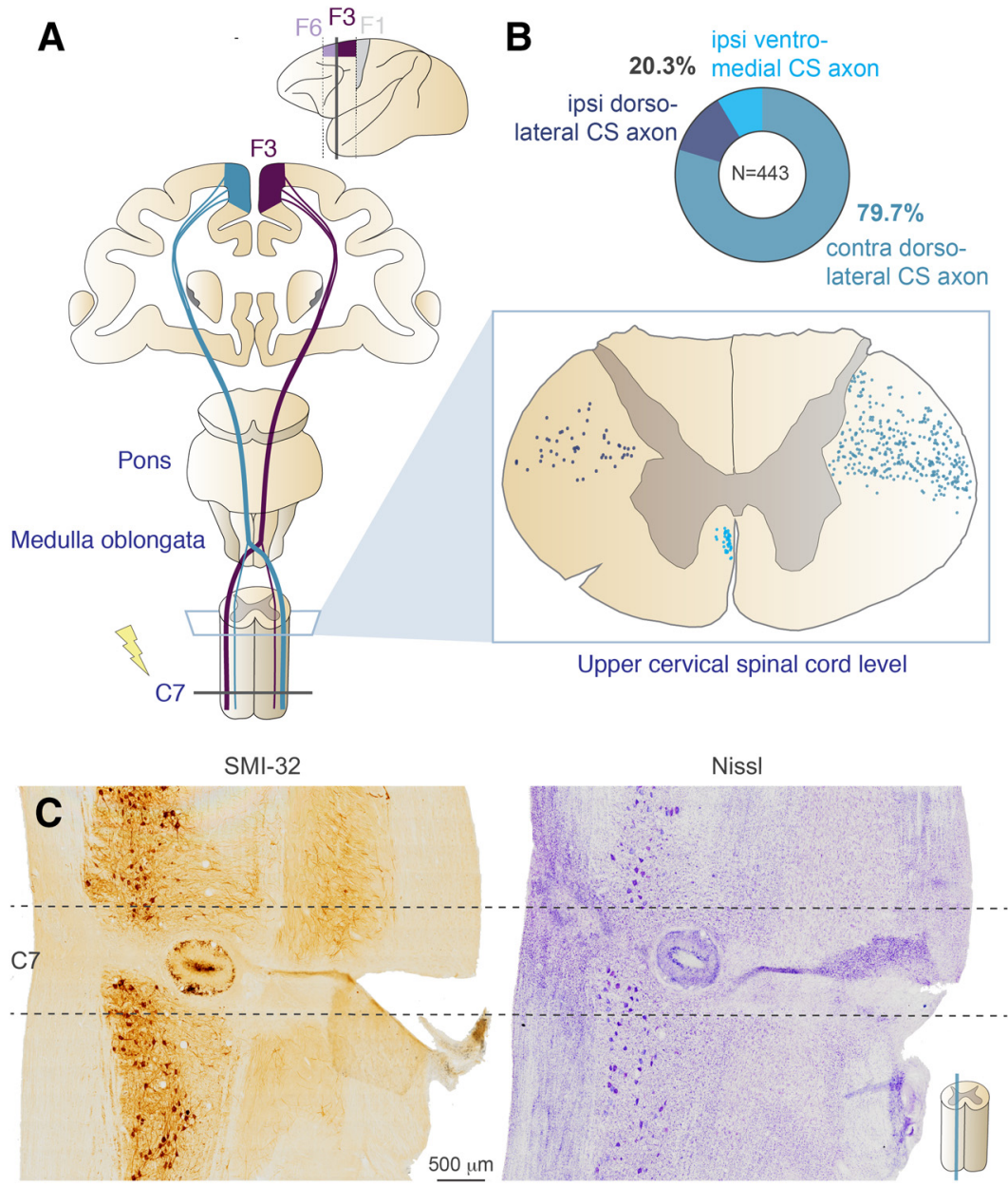
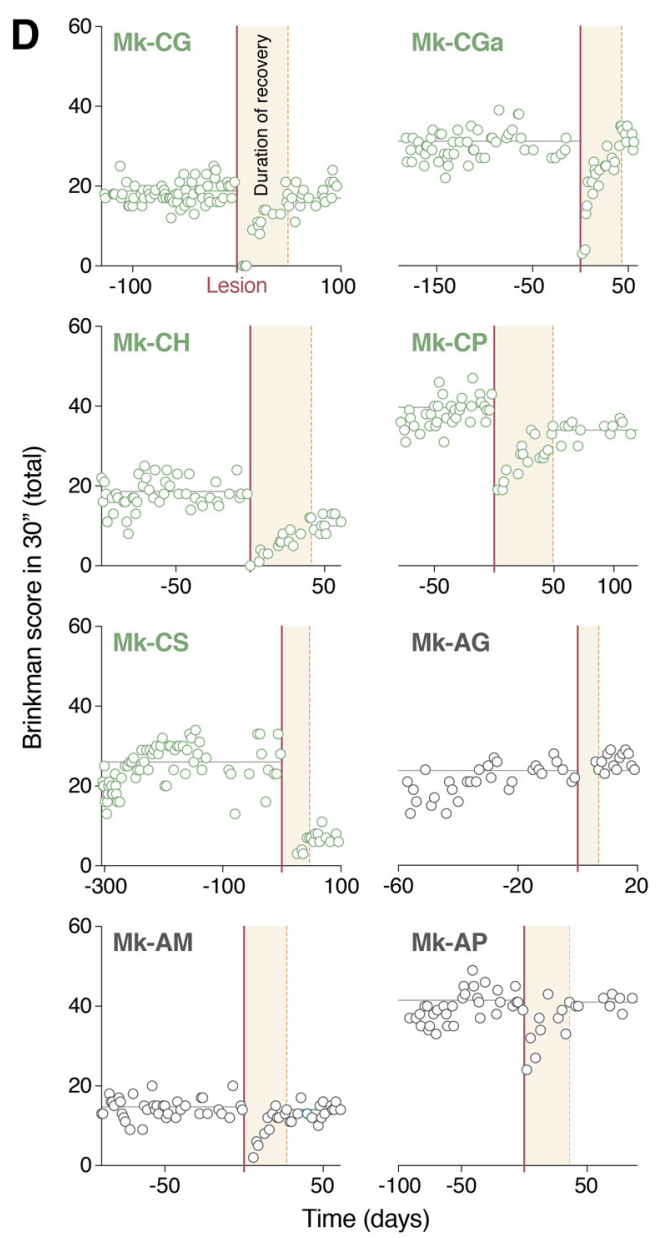

Figure 1. CS projection of SMA. A, upper panel, Schematic representation of a macaque brain showing the location of pre-SMA (area F6), SMA-proper (area F3), and M1. Lower panel, Schematic representation of the CS projections of F6 (bilateral). B, lower panel, Upper cervical spinal cord section showing the localization of CS axons labeled by a unilateral BDA injection in the SMA. Upper panel, Pie chart reporting the percentage of BDA-positive CS axons present in the ipsilateral ventromedial, ipsilateral dorsolateral, and contralateral dorsomedial part of the spinal cord. The majority of BDA positive CS axons are located in the contralateral dorsolateral part of the spinal cord. $\boldsymbol{C}$, Photomicrographs of sagittal histologic sections of the spinal cord of a lesioned animal (Mk-CS) showing the induced permanent lesion at the C7/C8 level. The histologic sections derived from two different series processed to visualize SMI-32 staining (left) or Nissl staining (right). Scale bar: $500 \mu \mathrm{m}$. $\boldsymbol{D}$, Graphical representation of behavioral performance in the modified Brinkman board task of the macaque monkeys included in this study ( $\mathrm{SCl}$ in green and $\mathrm{SCl}+$ anti-Nogo-A treatment in dark gray). The manual performance of the ipsilesional hand is given by the score (number of pellets retrieved in the first $30 \mathrm{~s}$ of the task from the randomly distributed wells) as a function of time (days) before and after a SCl. Day 0 corresponds to the day of the lesion (vertical red line). Prelesional and postlesional average of the scores are indicated with horizontal gray lines. The total duration of functional recovery of manual dexterity after lesion is given by the time interval between the lesion (red vertical line) and the onset of the postlesion plateau (brown area).

Figure Contributions: E. M. Rouiller and E. Schmidlin performed and supervised the experiments on the monkeys and analyzed the data.

concerned, the underlying mechanisms are multiple and complex, involving plasticity at spinal, subcortical and cortical levels, depending also on the time course of rehabilitative training (Galea and Darian-Smith, 1997; Freund et al., 2007; Nishimura et al., 2007; Nishimura and Isa, 2009, 2012; Zaaimi et al., 2012; Sugiyama et al., 2013; Isa and Nishimura, 2014; Chao et al., 2019). At cortical level in particular, following unilateral cervical lesion, the bilateral $\mathrm{M} 1$ areas are involved in the functional recovery at early stage whereas, at later stage, the contralesional M1 area plays a major role, together with the bilateral PM (Freund et al., 2007; Nishimura et al., 2007; Nishimura and
Isa, 2012; Isa and Nishimura, 2014). Moreover, a recent study identified two distinct cortical network dynamics that are implicated in the recovery of a unilateral SCl: the grasping-related intrahemispheric interactions from the contralesional PM to the contralesional M1, and motorpreparation-related interhemispheric interactions from the contralesional to ipsilesional PM (Chao et al., 2019).

What happens in SMA Layer V bilaterally after cervical hemisection and functional recovery? One may hypothesize that CS neurons in SMA also survive (as those in M1: see above), but their phenotype is likely to be modified (connectional diaschisis), in a variable manner depending 
Table 1: Summary of the individual properties of each monkey

\begin{tabular}{|c|c|c|c|c|c|c|c|c|c|c|c|c|c|}
\hline & & \multicolumn{4}{|c|}{ Intact } & \multicolumn{5}{|c|}{ Untreated } & \multicolumn{3}{|c|}{ Treated with anti-Nogo-A antibody } \\
\hline & & Mk-IC & Mk-IE & Mk-IR & $M k-I Z$ & Mk-CG & $\mathrm{MK}-\mathrm{CGa}^{3}$ & $\mathrm{Mk}-\mathrm{CH}$ & $\mathrm{Mk}-\mathrm{CP}$ & Mk-CS & $\mathrm{Mk}-\mathrm{AG}^{3}$ & Mk-AM & Mk-AP \\
\hline \multirow[t]{4}{*}{ General information } & Birthday & 17.07 .99 & 15.06 .96 & 02.02 .04 & 12.05 .96 & 20.02 .01 & 21.05 .03 & 20.02 .01 & 22.12 .97 & 30.04 .97 & 28.03 .02 & 20.02 .01 & 09.03 .98 \\
\hline & Sex & M & M & $\mathrm{F}$ & M & M & M & M & $\mathrm{F}$ & M & M & M & $\mathrm{F}$ \\
\hline & Species & Fasc & mul & Fasc & Fasc & fasc & fasc & fasc & fasc & mul & fasc & fasc & fasc \\
\hline & Date of killing & 17.08 .09 & 15.02 .02 & 22.12 .09 & 09.02 .04 & 13.01 .05 & 19.01 .07 & 07.02 .05 & 09.11 .04 & 21.09 .01 & 03.08 .05 & 14.02 .05 & 16.11 .04 \\
\hline \multirow[t]{7}{*}{ Lesion } & Date of lesion & - & - & - & - & 25.08 .04 & 16.08 .06 & 29.09 .04 & 04.05 .04 & 07.03 .01 & 13.04 .05 & 29.09 .04 & 02.06 .04 \\
\hline & SCl side & - & - & - & - & left & right & right & left & left & left & right & left \\
\hline & Weight at time of lesion (kg) & - & - & - & - & 5.1 & - & 4.1 & 3.8 & 4.0 & 3.7 & 4.5 & 4.2 \\
\hline & Age at the time of the lesion (d) & - & - & - & - & 1282 & 1183 & 1317 & 2325 & 1407 & 1112 & 1317 & 2277 \\
\hline & Hemisection extent (\%) & - & - & - & - & 51 & 73 & 90 & 45 & 63 & 78 & 80 & 58 \\
\hline & $\begin{array}{l}\text { Degree of functional recovery } \\
\text { from SCl, total score }(\%)^{1}\end{array}$ & - & - & - & - & 90 & 100 & 53 & 83 & 22 & 100 & 96 & 99 \\
\hline & Duration of functional recovery $(\mathrm{d})^{2}$ & - & - & - & - & 49 & 43 & 41 & 49 & 47 & 7 & 27 & 36 \\
\hline \multirow[t]{4}{*}{ Treatment } & type of antibody & - & - & - & - & ctrl & ctrl & ctrl & ctrl & ctrl & ATI & ATI & $11 \mathrm{c} 7$ \\
\hline & Cocentration $(\mathrm{mg} / \mathrm{ml})$ & - & - & - & - & 9 & 7 & 9 & 3.7 & 3.7 & 3.6 & 9 & 3.7 \\
\hline & Volume (ml) & - & - & - & - & 4 & 2 & 4 & 4 & 4 & 2 & 4 & 4 \\
\hline & Days of antibody injection & - & - & - & - & 30 & 29 & 28 & 29 & 32 & 28 & 28 & 15 \\
\hline
\end{tabular}

$\mathrm{M}=$ male; $\mathrm{F}=$ female; fasc $=M$. fascicularis; $\mathrm{mul}=\mathrm{M}$. mulatta; rhe $=$ Rhesus.

${ }^{1}$ Expressed in percentages of postlesion total score at plateau divided by prelesion total score in the modified Brinkman board task: all slots.

2 Time interval from the day of lesion to the beginning of postlesion plateau, as defined by Kaeser et al. (2011).

${ }^{3} \mathrm{Mk}-\mathrm{CGa}$ and Mk-AG suffered from a more caudal lesion than C7/C8. These monkeys were taken into account in this study but with some reserves.

on the hemisphere (because of the asymmetric strength of its CS projection), as well as on lesion parameters, such as lesion extent, as well as on functional recovery parameters (duration of recovery, extent of recovery). To test these different hypotheses, SMI-32-labeled neurons in the bilateral SMAs were analyzed histologically in eight macaque monkeys subjected to cervical hemisection and compared with intact monkeys $(n=4)$, based on the assumption that graded changes in the phenotype of CS neurons in SMA impacts on the detection of those neurons using the marker SMI-32 (Contestabile et al., 2018; see also Geyer et al., 2000).

\section{Materials and Methods}

The methods to analyze the histologic sections in SMA following $\mathrm{SCl}$ were similar to those reported in detail in a recently published open access article (Contestabile et al., 2018), also focused on SMA but following a lesion of the M1. Nevertheless, for the sake of convenience, the methods are reminded here, although in a somewhat shorter version.

\section{Macaque monkeys}

The present histologic analysis in SMA was conducted on 12 adult macaque monkeys (10 Macaca fascicularis, two Macaca mulatta; Table 1). All procedures were conducted in accordance with guidelines of the federal and local (cantonal) veterinary authorities (veterinary authorizations: 157e/04, 175/04, 187/05, 188/06, 193/07). All 12 monkeys included in the current study were already reported in previous articles addressing distinct issues related to C7 spinal cord lesion (Freund et al., 2006, 2007, 2009; Beaud et al., 2008, 2012; Hoogewoud et al., 2013) or as intact control animals (Contestabile et al., 2018). Eight animals (Mk-CG, Mk-CGa, Mk-CH, Mk-CP, Mk-CS, Mk-AG, Mk-AM, and Mk-AP) were subjected to a unilateral cervical cord lesion at C7 level, for whom detailed and comprehensive experimental data were reported previously, such as lesion characteristics, behavior, plasticity of CS projection system in M1, and at spinal cord level, effects of the anti-Nogo-A antibody therapy (Freund et al., 2006, 2007, 2009; Beaud et al., 2008, 2012; Hoogewoud et al., 2013). Four other monkeys (Mk-IC, Mk-IE, Mk-IR, and $\mathrm{Mk}-\mathrm{IZ}$ ) had no lesion (intact monkeys) and were used as controls; they already appeared as such in a recent study (Contestabile et al., 2018). Three out of eight cervical cord lesioned monkeys were treated with anti-Nogo-A antibody (Mk-AG, Mk-AM, and Mk-AP). Comprehensive descriptions of the anti-Nogo-A antibody treatment procedure were published earlier (Freund et al., 2006, 2007, 2009; Beaud et al., 2008, 2012; Hoogewoud et al., 2013). In short, the anti-Nogo-A antibody treatment $(14.8 \mathrm{mg})$ was applied during for four weeks immediately after the SCI. The anti-Nogo-A antibody was delivered from an osmotic pump, placed in the back of the animal. The other five monkeys subjected to $\mathrm{SCl}$ received a control antibody (Freund et al., 2006, 2007, 2009). At the end of the behavioral assessments (see below), the animals were euthanized under deep anesthesia obtained with an intraperitoneal overdose of pentobarbital sodium $(90 \mathrm{mg} / \mathrm{kg}$ body weight), as previously reported (Freund et al., 2006, 2007, 2009; Beaud et al., 2008, 2012; Hoogewoud et al., 2013).

\section{Behavior}

As recently reported (Contestabile et al., 2018), manual dexterity was quantified in the eight monkeys subjected to unilateral $\mathrm{SCl}$, based on the modified Brinkman board task, testing the precision grip (opposition of thumb and index finger), needed to grasp small food pellets in 25 vertically oriented slots and 25 horizontally oriented slots, randomly positioned over a Perspex board (Schmidlin et al., 2011). After habituation to the housing facility and transfer to a primate chair using positive reinforcement 
(http://www.unifr.ch/neuro/rouiller/home/nhp), the macaques monkeys were progressively trained daily to perform the modified Brinkman board task, separately for each hand, until they reached a prelesion plateau of performance. The duration of the training period and the plateau level of performance was variable across monkeys, as reported earlier based on a large cohort of animals (Kaeser et al., 2014). Then, at plateau, the monkeys were tested daily, once for each hand, during a prelesion phase of several weeks to establish a score of manual dexterity of reference in the intact monkey. The same behavioral test procedure was pursued also daily after the $\mathrm{SCl}$, without additional training besides the test itself. Based on the postlesion tests, it was possible to assess the progressive functional recovery from the $\mathrm{SCl}$ and the performance level of the postlesion plateau.

The dramatic drop of score (usually to zero) immediately after the lesion and the deficits of precision grip following such cervical lesion have been reported in detail earlier (Freund et al., 2006, 2007, 2009; Hoogewoud et al., 2013) and are summarized in Figure $1 D$. After unilateral $\mathrm{C} 7$ injury, a progressive although incomplete functional recovery was observed until reaching a plateau of motor performance a few weeks after the SCI (Freund et al., 2006, 2007, 2009; Hoogewoud et al., 2013; Fig. 1D). The behavioral parameter of interest here was the percentage of functional recovery for the ipsilesional hand, given by the average total score (number of pellets retrieved from both vertical and horizontal wells in $30 \mathrm{~s}$ ) at postlesion plateau divided by the average total score at prelesion plateau (Freund et al., 2006, 2007, 2009; Hoogewoud et al., 2013; Fig. 1D). Moreover, the plots of manual dexterity scores as a function of time were used to define the time duration until the postlesion plateau was reached (duration of functional recovery; Fig. 1D).

\section{Unilateral cervical cord lesion}

The surgical procedure to perform the unilateral lesion was described in detail in previous reports (Freund et al., 2006, 2007, 2009; Beaud et al., 2008, 2012; Hoogewoud et al., 2013), an experimental procedure summarized below. Anesthesia was induced by an intramuscular injection of ketamine (Ketalar; Parke-Davis; $5 \mathrm{mg} / \mathrm{kg}$ ), and atropine was injected intramuscularly $(0.05 \mathrm{mg} / \mathrm{kg})$ to reduce bronchial secretions. Before surgery, the analgesic Carprofen was delivered (Rymadil, $4 \mathrm{mg} / \mathrm{kg}$, s.c.). Then, deep and stable anesthesia was obtained via a continuous perfusion $(0.1 \mathrm{ml} / \mathrm{min} / \mathrm{kg})$ through an intravenous catheter placed in the femoral vein of a mixture of $1 \%$ propofol (Fresenius) and a $4 \%$ glucose solution. The monkey was positioned in a stereotaxic headholder in a ventral decubitus position, allowing the spinal processes from C2 to Th1 to be exposed, preceding a complete C6 laminectomy and an upper $\mathrm{C} 7$ hemilaminectomy. The dorsal root entry zone at the C7/C8 border was then identified, providing a medial landmark for placing a surgical blade (no. 11; Paragon), which was inserted vertically $4 \mathrm{~mm}$ in depth to generate an incomplete section of the cervical cord at C7 level. In most cases, such a section completely interrupted the CS tract in the dorsolateral funiculus unilaterally. Following the $\mathrm{SCl}$, the monkey was kept alone in a separate cage for a couple of days to monitor its health condition and provide specific postoperative care (antibiotics, analgesics).

\section{Histology and neuroanatomical material for analysis}

The general histologic and analysis methods are similar to those recently reported (Contestabile et al., 2018) and repeated in a shorter version below. After euthanasia, the spinal cord segment (C3-T4) comprising the SCl was cut parasagittally in either three or five series of, respectively, 50 or $30 \mu \mathrm{m}$ sections and processed to visualize either BDA, Nissl or SMI- 32 staining (Fig. $1 A-C)$. The extent of the cervical cord lesion was measured in consecutive longitudinal sections (scar tissue and absence of neuronal staining) and transposed in a transversal reconstruction to assess the percentage of lesion with respect to the entire corresponding hemicervical cord (white and gray matter).

In all monkeys ( $\mathrm{SCl}$ and intact), the brain was cut in the frontal plane into $50-\mu \mathrm{m}$-thick frozen sections with both hemispheres facing each other on the same slide (Fig. $2 A)$. Sections were collected in five or eight consecutive series, one of them immunoreacted to visualize the marker SMI-32 (Sternberger and Sternberger, 1983), as previously reported (Liu et al., 2002; Wannier et al., 2005; Beaud et al., 2008). Frontal brain sections were examined under bright-field illumination (at a total magnification of $200 \times$ ). Brain regions of interest (mostly SMA) were vectorized using Neurolucida 9 (MBF Bioscience) with a computer-interfaced Olympus BX40 microscope (Olympus Schweiz AG), a computer-controlled motorized stage (Märzhäuser Wetzlar GmbH, type EK $3275 \times 50$ ) and a digital camera (Olympus U-PMTVC). The Photomicrographs captured with a digital camera were processed using the CorelDraw software (color, brightness, and contrast were not modified) and then quantification was conducted using the software Neuroexplorer (MBF Bioscience; for example, see Fig. $2 B-D$ ). At that step, the investigator of the histologic material was blinded against information on animal group (intact vs SCl) and side of the cervical cord hemisection. The size of the $\mathrm{SCl}$ expressed as a percentage of hemisection extent were reported previously (Freund et al., 2006, 2007, 2009; Beaud et al., 2008, 2012; Hoogewoud et al., 2013) and are reminded in Table 1. SMA and its two subdivisions (F3 and F6, respectively, corresponding to SMA-proper and pre-SMA) in both hemispheres was delineated from M1 or PM laterally and from cingulate motor area (CMA) ventrally, based on cytoarchitectural landmarks (Liu et al., 2002). SMI-32-positive neurons were counted in SMA Layers $V$, and the surface of the delineated F3 or F6 was calculated in relation with the defined limits. Based on an exhaustive plotting method (Fregosi and Rouiller, 2017; Fregosi et al., 2017, 2018), the cellular density in Layer V of F3, respectively, F6, was computed on individual sections. It is the number of identified SMI-32-positive cells in that layer in one hemisphere divided by the corresponding volume of SMA (given by the thickness of the section multiplied by the area-of-interest). The criteria used to include a neuron were the same proposed in our previous article 

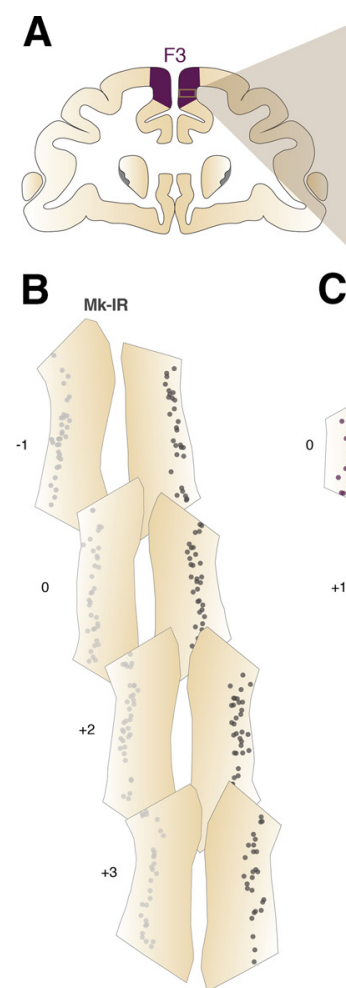

C Mk-CG
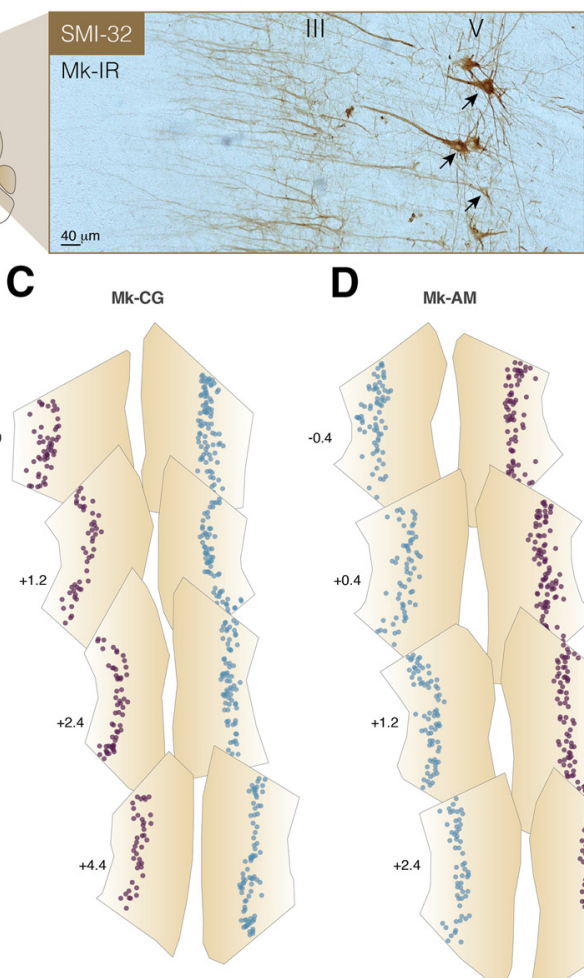

D Mk-AM

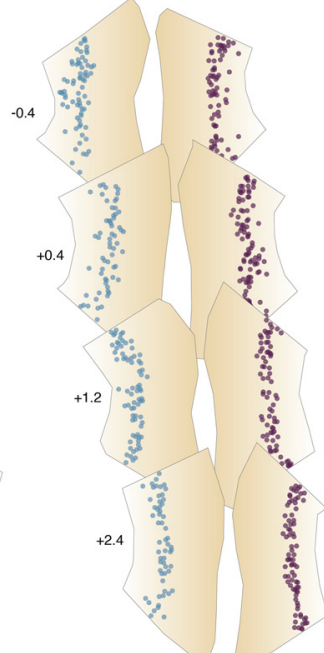

Neurons in: - left hemisphere, - right hemisphere, - ipsilesional hemisphere, - contralesional hemisphere

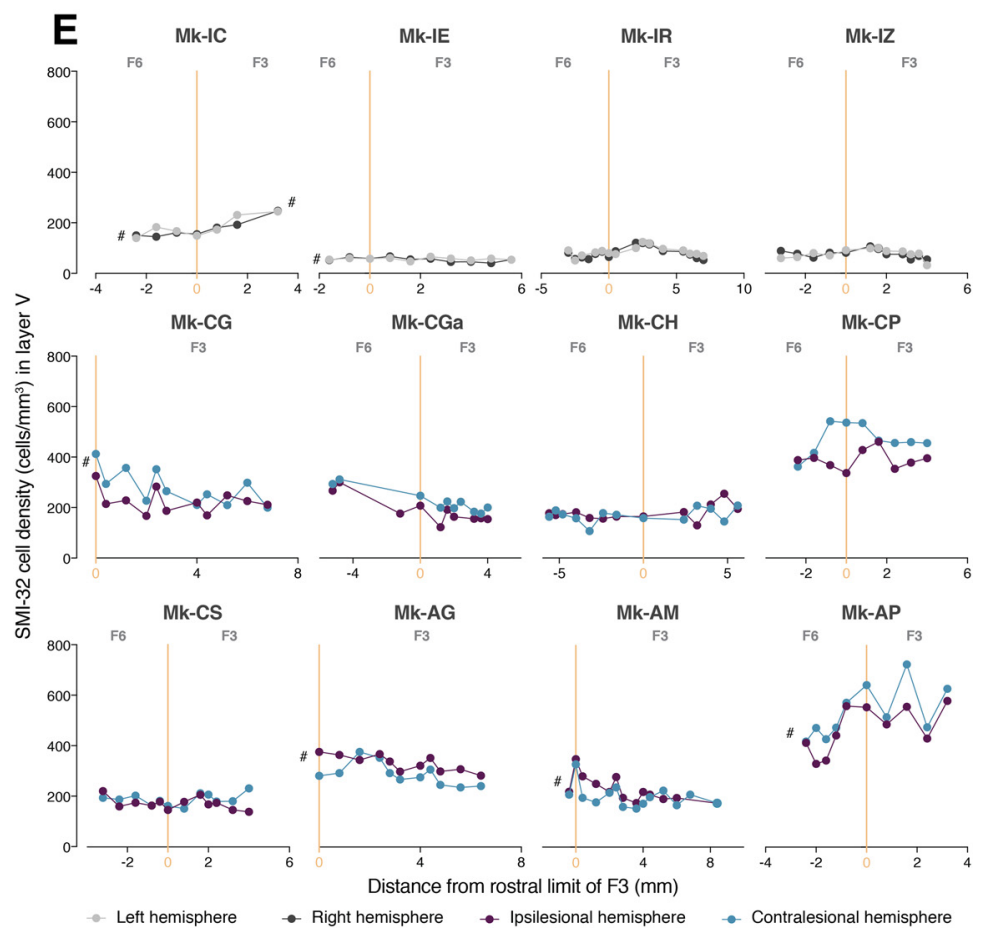

Figure 2. Neuronal density in the Layer $\mathrm{V}$ of SMA in intact and SCl monkeys. A, Photomicrograph of coronal brain histologic section of an intact macaque monkey (Mk-IR) stained with SMI-32 and magnified in F3 (scale bar: $40 \mu \mathrm{m}$ ). Layers III and V are visible and SMI-32-positive pyramidal neurons are indicated with arrows. $\boldsymbol{B}-\boldsymbol{D}$, Localization of SMI-32-positive Layer $\mathrm{V}$ neurons in bilateral F3 and F6 areas of three representative macaque monkeys (intact: Mk-IR, SCl: Mk-CG and SCl + anti-Nogo-A treatment: Mk-AM). Four sections per macaque monkeys are shown and the relative rostro-caudal position of the sections from the F3-F6 border is reported in millimeters. Negative distance values belong to F6 and positive distance values belong to F3. $E$, Graphs representing the rostro-caudal gradient (from F6 to F3) of SMI-32-positive cell density in Layer V of all monkeys (intact: Mk-IC, Mk-IE, Mk-IR, and Mk-IZ; SCl: Mk-CG, Mk-CGa, Mk-CH, Mk-CP, and Mk-CS; SCI + anti-Nogo-A treatment: Mk-AG, Mk-AM, and Mk-AP). The cell density for each hemisphere is plotted as a function of the distance from the F3-F6 border, which has been set to $3 \mathrm{~mm}$ rostrally to 
continued

the genu of the arcuate sulcus. Negative distance values belong to F6 and positive distance values belong to F3. The symbol \# was used to indicate that the analyzed cortex region was not complete (sections lacking for the analysis).

Figure Contributions: E. M. Rouiller and E. Schmidlin performed the experiments on the monkeys and generated the histologic sections. A. Contestabile and R. Colangiulo performed the microscopic analysis of the histologic sections. A. Contestabile analyzed the data.

(Contestabile et al., 2018): (1) SMI-32-positive; (2) the soma, the nucleus or the nucleolus and at least four proximal dendrites of the neuron have to be identifiable; and (3) the neuron is located in the cortical Layer V. The criteria to set the rostrocaudal limit between F3 and F6, as well as the precise territories of $\mathrm{F} 3$ and $\mathrm{F} 6$ analyzed were the same as in Contestabile et al. (2018).

\section{Interhemispheric difference of cell density (IDCD)}

An IDCD in SMA Layer $V$ was computed in each histologic section by subtracting the SMI-32-positive neuron density in the ipsilesional SMA (relatively intact side) from the SMI-32-positive neuron density in the contralesional SMA (strongly connected with the SCl; Fig. 3B,D). Positive IDCD means that more SMI-32 neurons were found in SMA strongly connected to the side where the $\mathrm{SCl}$ was performed, whereas negative IDCD corresponds to a larger number of SMI-32 neurons in Layer V of SMA homolateral to the SCI. In intact monkeys, IDCD was expected to be close to zero, whereas in monkeys subjected to $\mathrm{SCl}$, the IDCD was hypothesized to diverge from zero (Fig. 5A, e.g., gray line). As for our previous work (Contestabile et al., 2018), the interhemispheric comparison was made on each individual section, so that the mirrored SMA territories analyzed on each side had comparable area and position.

\section{Single SMI-32-labeled neuron analysis}

To investigate whether the unilateral $\mathrm{SCl}$ impacted on the microstructure of single SMI-32-positive neurons in SMA Layer V, in particular on their dendritic trees, a socalled Sholl analysis was performed, identical to the one reported recently (Contestabile et al., 2018). The sampling and inclusion criteria were similar: three SMI-32-positive pyramidal neurons of Layer $\mathrm{V}$ per hemisphere per section were analyzed in four different sections (12 neurons per hemisphere in total) in each of five representative monkeys: two intact monkeys and three monkeys with SCl. The following criteria were applied to specifically select SMI-32-positive neurons: (1) to be representative of the entire region-of-interest, one neuron was picked in each of the dorsal, middle, and ventral parts of the medial wall in F3; and (2) at least intact primary and secondary dendrites and an apical dendrite reaching the Layer III without interruption had to be clearly identified. Two distinct regions of the dendritic tree of each SMI-32-positive neurons were analyzed: the basal dendrites excluding the axon and the apical dendrite.

\section{Statistics}

Statistical analysis was conducted with GraphPad Prism 7. The normality of sample distributions was assessed with the Shapiro-Wilk criterion. In each monkey, the statistical significance of IDCDs of SMI-32-positive neurons between the ipsilesional and contralesional hemispheres was assessed using a paired $t$ test or a Wilcoxon test (according to the data distribution) as the neuronal density was directly compared across the two hemispheres on the same section. In order to compare the interhemispheric morphologic data and the results of the Sholl analysis, a two-way repeated-measures ANOVA with Bonferroni's post hoc test correction wsd used $(* p=$ $0.05, * * p=0.01, * * * p=0.001, * * * * p=0.0001$ ). Data are represented as the mean \pm SEM, and the significance was set at $95 \%$ of confidence.

\section{Results}

Based on a previously available case (Rouiller et al., 1996; intact monkey 93-81), subjected to a unilateral injection of the anterograde tracer BDA in the SMA (F3, focused to the hand area), the bilateral distribution of CS axons was established at cervical level. The majority of CS projection originating from SMA crossed the midline (79.7\%), whereas only $20.3 \%$ of the BDA positive CS axons were located on the ipsilateral side of the cervical cord $(11.7 \%$ in the dorsolateral part and $8.6 \%$ in the ventromedial part; Fig. 1A,B). These results suggest that a unilateral cervical cord injury (SCl; Fig. $1 C$ ) retrogradely impacts more on the contralesional SMA than on the ipsilesional one (connectional diaschisis).

As previously reported (Freund et al., 2006, 2007, 2009; Beaud et al., 2008, 2012; Hoogewoud et al., 2013), eight macaques monkeys (Table 1) were unilaterally injured at C7 level (SCl animals: Mk-CG, Mk-CGa, Mk-CH, Mk-CP, and Mk-CS; SCI + Anti-Nogo-A animals: Mk-AG, Mk-AM, and $M k-A P)$, and the manual dexterity was quantified using the modified Brinkman board task. After unilateral C7 injury, the ipsilesional manual dexterity was severely affected, followed by a progressive although incomplete functional recovery, reaching a plateau of motor performance a few weeks after the SCI (Fig. 1D). Thanks to this longitudinal quantification of the manual dexterity, it was possible to calculate the extent of functional recovery (in $\%$ ) of the ipsilesional hand and the time duration needed to reach the postlesional plateau (duration of functional recovery; Table 1; Fig. 1D).

\section{Neuronal density in the Layer V of SMA after a SCI}

In order to quantify the effect of a unilateral SCI (Fig. 1C) on SMA Layer $V$ pyramidal neurons, SMI-32-labeled neurons in the bilateral F3 (SMA-proper) and F6 (preSMA) were analyzed histologically in eight macaque monkeys subjected to cervical hemisection and compared with intact monkeys $(n=4)$. First, we quantified the density of SMI-32-positive Layer $\mathrm{V}$ neurons in the delimited F3 and $\mathrm{F} 6$ areas in both hemispheres along the rostro-caudal 
F3 - layer V
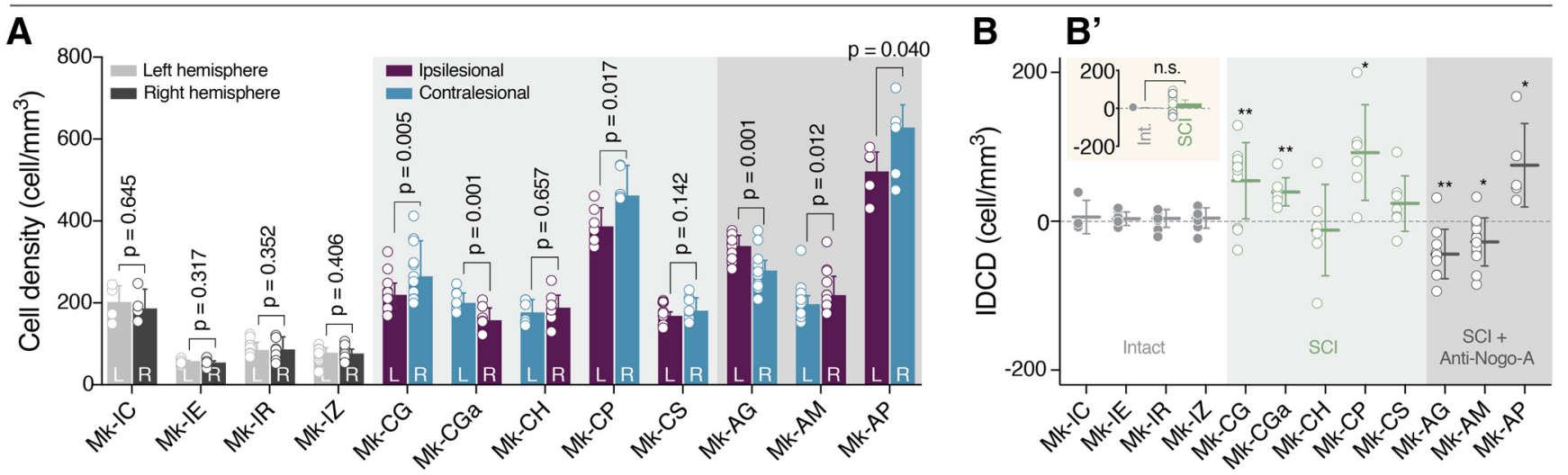

\section{F6 - layer V}
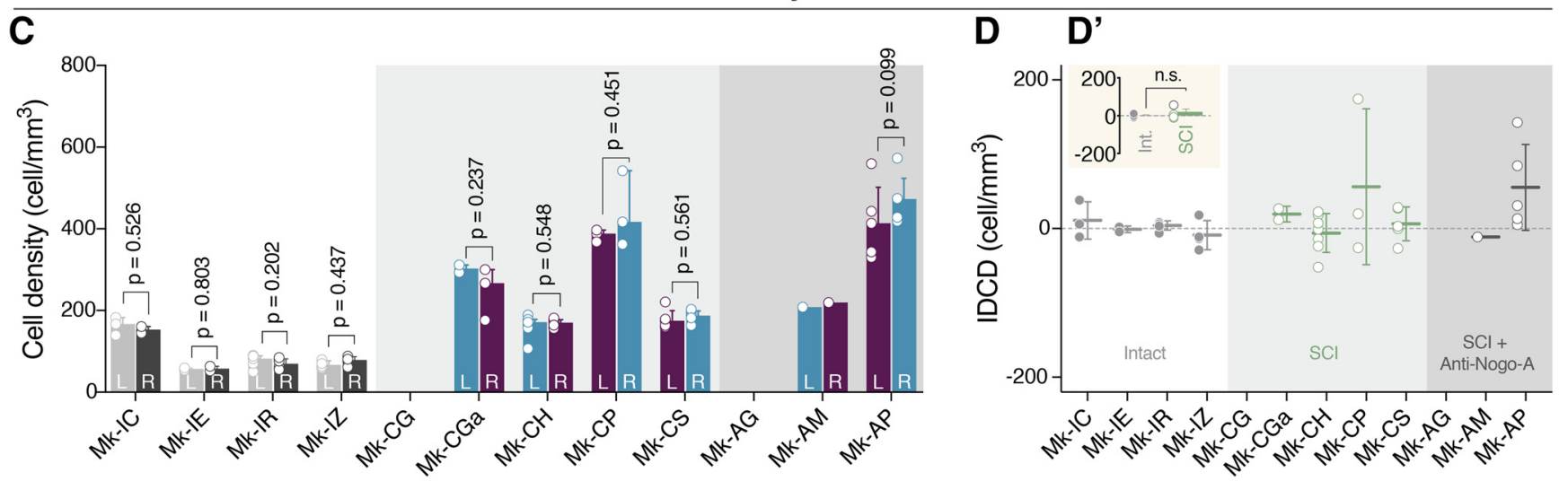

Figure 3. IDCD after SCI in F3 and F6. A, Histograms reporting the cell density of Layer V SMI-32-positive neurons in F3 for intact (white background), SCI (light gray background), and SCl-treated monkeys (dark gray background). For each couple of histograms are reported the side $(\mathrm{L}=$ left and $\mathrm{R}=$ right) and the location in function of the SCI (Ipsi = ipsilesional and Contra = contralesional). As statistical test, a paired $t$ test or Wilcoxon test was performed ( $p$ values are reported) comparing the cell density in the two hemispheres in each consecutive histologic section. $\boldsymbol{B}$. Histograms showing the IDCD of Layer V SMI-32-positive neurons of F3 for intact (white background), SCI (light gray background), and SCl-treated monkeys (dark gray background). A positive IDCD corresponds to an ipsilesional bias in pyramidal cell density, while a negative IDCD corresponds to a contralesional bias in pyramidal cell density. $\boldsymbol{B}^{\prime}$, upper left inset, Comparison of calculated IDCD in F3 between groups. No statistical difference is observed (unpaired $t$ test: $t_{(10)}$ $=0.8276, p=0.4272$ ). $\boldsymbol{C}$, Histograms reporting the cell density of Layer V SMI-32-positive neurons in F6 for intact (white background), SCl (light gray background), and SCl-treated monkeys (dark gray background). For each couple of histograms, are reported the side ( $\mathrm{L}=$ left and $\mathrm{R}=$ right) and the location in function of the $\mathrm{SCl}$ (Ipsi = ipsilesional and Contra = contralesional). As statistical test, a paired $t$ test or Wilcoxon test was performed ( $p$ values are reported) comparing the cell density in the two hemispheres in each consecutive histologic section. $\boldsymbol{D}$, Histograms showing the IDCD of Layer V SMI-32-positive neurons of F6 for intact (white background), SCl (light gray background), and SCl-treated monkeys (dark gray background). D', upper left inset, Comparison of calculated IDCD in F6 between groups. No statistical difference is observed (unpaired $t$ test: $t_{(7)}=1.084, p=0.3142$ ). For $\boldsymbol{A}$, $\boldsymbol{C}$, the median and the interquartile range are indicated. For $\boldsymbol{B}, \boldsymbol{D}$, the mean $\pm \mathrm{SD}$ are reported. $\mathrm{L}=$ left hemisphere, $\mathrm{R}=$ right hemisphere, $* p \leq 0.05, * * p \leq 0.01)$.

Figure Contributions: E. M. Rouiller and E. Schmidlin performed the experiments on the monkeys and generated the histologic sections. A. Contestabile and R. Colangiulo performed the microscopic analysis of the histologic sections. A. Contestabile analyzed the data.

axis in coronal sections (Fig. 2A-D). In F6 and F3 Layer V, the SMI-32-positive neurons' densities ranged approximately from 50 cells $/ \mathrm{mm}^{3}$ to 250 cells $/ \mathrm{mm}^{3}$ across sections/hemispheres/monkeys in the intact group, whereas in the lesioned monkeys, SMI-32-positive neurons' densities in SMA Layer $V$ ranged approximately from 100 to $700 \mathrm{cells} / \mathrm{mm}^{3}$ (Fig. 2E). With respect to those ranges of cellular densities, in the $\mathrm{SCl}$ group, there is no obvious difference between the anti-Nogo-A antibody-treated monkeys $(n=3)$ and the control antibody treated animals $(n=5)$. In addition, the range of SMI-32-positive neurons' densities in the SMA of SCl animals seemed to reach higher values than the intact group (in fact higher than three intact monkeys with low cellular densities in F3, whereas the fourth intact monkey exhibits a cellular density comparable to five monkeys of the $\mathrm{SCl}$ group). However, the very large interindividual variability of the histologic staining quality precludes interindividual comparison between the two groups of monkeys and strongly affects the absolute quantification of neuronal density. The quality of SMI-32 staining in three of the four intact monkeys may have been different from in the SCl 
monkeys, leading to the differences in the absolute numbers of SMI-32-positive neurons between the two groups. On the other hand, the quality of staining does not interfere when the comparison is intraindividual and furthermore restricted to a single histologic section. For this reason, we performed a direct comparison between the hemispheres (left hemisphere compared with the corresponding right hemisphere on the same section).

\section{IDCD}

As expected, intact monkeys presented no significant difference in their IDCDs for the SMI-32-positive neurons in Layer V of F3 (Fig. $3 A, B$ ). On the other hand, the IDCDs of SMI-32-positive neurons were significantly different in six out of eight monkeys subjected to SCl (Fig. 3B). However, the IDCD was not systematically biased toward the same hemisphere (ipsilesional vs contralesional; Fig. $3 B$ ). Four lesioned monkeys (Mk-CG, Mk-CGa, Mk-CP, and Mk-AP) exhibited a significantly higher contralesional density of SMI-32-positive neurons in F3 Layer V, corresponding to a positive IDCD, while two SCI monkeys (Mk$A G$ and Mk-AM) had a significantly higher ipsilesional density of SMI-32-positive neurons in F3 Layer V, corresponding to a negative IDCD (Fig. $3 A, B$ ).

In order to have an internal control, the cellular density of SMI-32-positive neurons was also quantified in the Layer V of F6 (pre-SMA), a motor cortical area lacking CS neurons (Luppino et al., 1994). The assessment of cellular density in F6 Layer V showed no significant IDCD, both in intact animals and in $\mathrm{SCl}$ animals (Fig. 3C,D). This observation indicates that SMI-32-positive neurons in F6 Layer $\checkmark$ were not affected by the $\mathrm{SCl}$, as expected.

Finally, we did not observe significant difference between the calculated IDCD between intact and injured animals neither in F3 nor in F6 (Fig. 3B', $D^{\prime}$ ).

\section{Arborization of Layer V SMI-32-positive neurons in F3 after $\mathrm{SCl}$}

Microstructural changes of the basal (Fig. 4A) and apical dendritic (Fig. 4G) arborization of SMI-32-positive neurons located in Layer V of F3 was assessed based on a Sholl analysis conducted in two control animals (Mk-IR and $\mathrm{Mk}-\mathrm{IE}$ ) and in three representative lesioned monkeys (Mk-CGa, Mk-CP, and Mk-AG). For all five animals, the Sholl analysis yielded a classical inverted U-shape curve with a tail on the right, corresponding to an increase of dendritic intersection numbers going away from the soma up to a peak, followed by a comparable progressive decrease ending with slower fading at larger distances from the soma. In most analyzed monkeys, the number of dendritic intersections peaked at a distance of $\sim 50 \mu \mathrm{m}$ from the soma, both for basal and apical dendrites (Fig. 4). As excepted, no interhemispheric difference was observed in the two control monkeys for both the basal (Fig. 4B,C) and apical dendrites (Fig. $4 H, I$ ). After SCl, two out of three monkeys exhibited in $\mathrm{F} 3$ interhemispheric differences in the basal dendritic arborization (Fig. 4D-F). In fact, for Mk-CP and Mk-AG, we observed the same interhemispheric bias consistent with the IDCD bias already observed for Layer $\mathrm{V}$ in $\mathrm{F} 3$ (Figs. 3B, 4E,F) for the basal dendrites. A comparable tendency (not statistically significant) was observed for Mk-CGa, the third representative lesioned animal. This significant interhemispheric difference of basal dendritic arborizations was verified within a limited distance range from the soma, going from 40 to $80 \mu \mathrm{m}$ (Fig. 4E,F). Interestingly, and in contrast to the basal dendrites, the apical dendrites exhibited clearly less pronounced interhemispheric difference in their numbers of dendritic intersections (Fig. $4 J-L$ ).

\section{Relationship of IDCDs in F3 with percentage of hemisection extent, duration and extent of functional recovery after $\mathrm{SCI}$}

Are the morphologic interhemispheric changes on SMI32-positive neurons in F3 Layer V induced by SCl (Figs. 2, 3 ) related to the characteristics of the lesion (size) and/or the properties of functional recovery? To this aim, IDCDs of SMI-32 neurons in F3 were plotted as a function of lesion extent (percentage of hemisection), durations of functional recovery and extent in percent of functional recovery (Fig. 5A-C). Interestingly, IDCDs in F3 Layer V were significantly inversely correlated with the size of the $\mathrm{SCl}$, expressed as the percentage of hemisection $(R=$ $-0.857, p=0.006$; Fig. $5 A$ ), independently of the presence/absence of anti-Nogo-A antibody treatment. A similar inverse correlation was found between IDCDs and lesion size in $\mathrm{F} 3$ after unilateral M1 lesion (Contestabile et al., 2018). In both cases, these correlation data indicate that a small lesion (ipsilateral in the case of cortical lesion or contralateral in case of $\mathrm{SCl}$ ) is associated with a largely positive IDCD, whereas large lesions are associated to negative IDCDs.

The relationship between the median IDCD values and the duration of functional recovery from $\mathrm{SCl}$ (Fig. 5B) was then investigated, based on behavioral data derived from the modified Brinkman board task: the IDCDs in F3 Layer $V$ were correlated with the duration of functional recovery $(R=0.734, p=0.038$; Fig. $5 B)$, again independently of the presence/absence of treatment. Interestingly, there was no statistically significant correlation between the duration of functional recovery from $\mathrm{SCl}$ and the hemisection extent of the spinal cord ( $R=-0.525, p=0.181$; Fig. $5 C$ ). However, the three $\mathrm{SCl}$ monkeys which received the antiNogo-A antibody treatment recovered faster (shorter time to reach the postlesional plateau) than the control antibody-treated monkeys, a difference already visible in Figure 5B. In contrast to the parameters lesion extent and duration of functional recovery, IDCDs in F3 Layer $\mathrm{V}$ were not correlated with the parameter extent of functional recovery (expressed in \%; $R=0.054, p=0.889$; Fig. $5 D$ ), assessed with the total score of pellets retrieval in the modified Brinkman board task. Finally, as observed in Contestabile et al. (2018); no correlation was observed between the percentage of functional recovery in the modified Brinkman board task and duration of functional recovery $(R=0.176, p=0.301$; Fig. $5 E)$, suggesting that a longer duration of functional recovery did not mean a better functional recovery. 
A

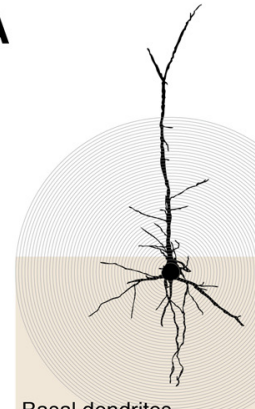

Basal dendrites

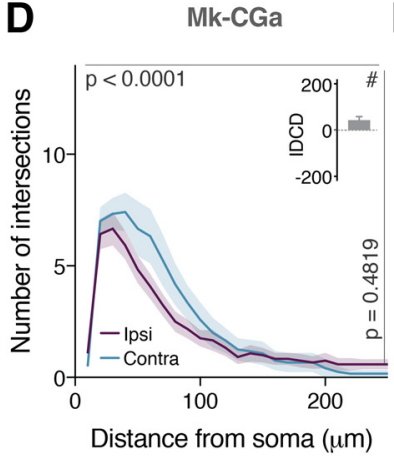

B

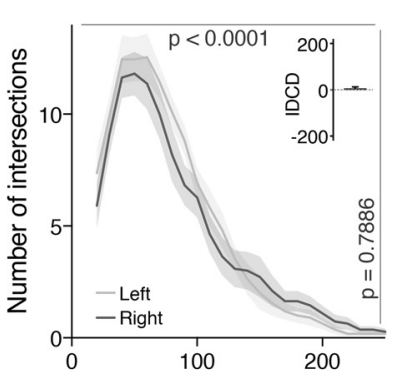

E

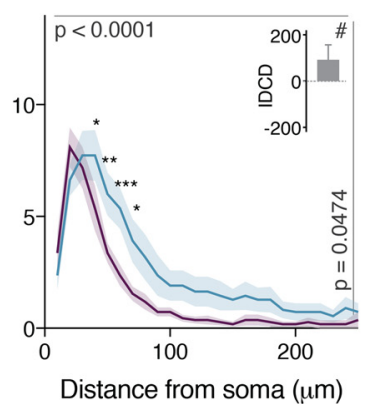

C

Mk-IR

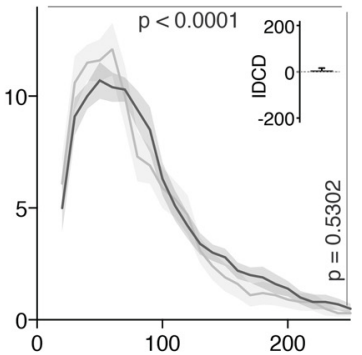

$\mathbf{F}$
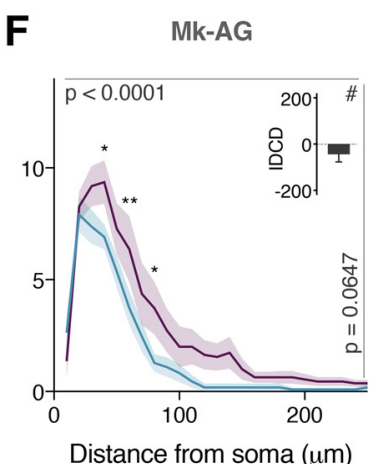

G

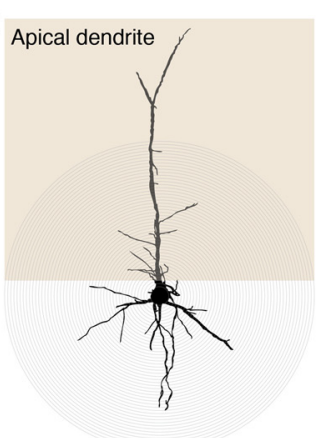

J

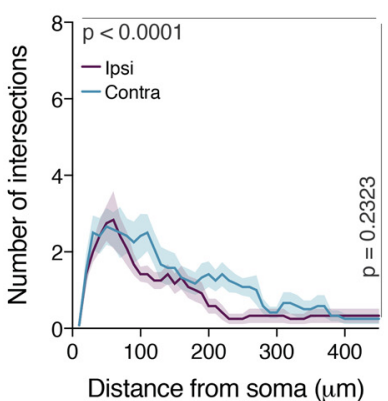

H

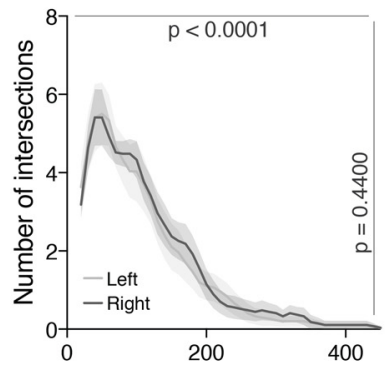

K
Mk-CP

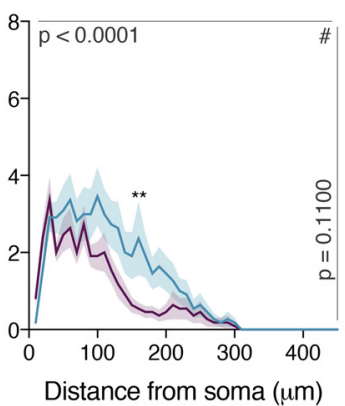

Mk-IR

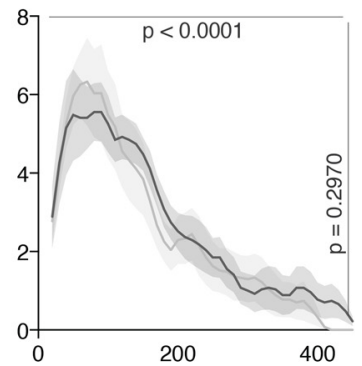

Mk-AG

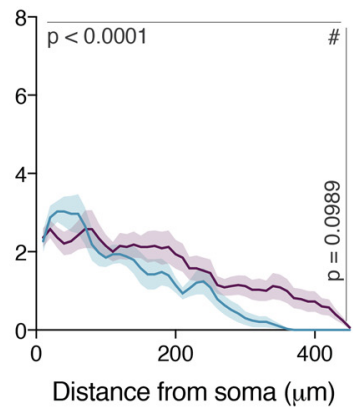

Figure 4. Arborization of Layer V SMI-32-positive neurons in F3 after a SCl. $\boldsymbol{A}$, Neuronal reconstruction example of basal dendrites of a Layer V SMI-32-positive neuron. $\boldsymbol{B}-\boldsymbol{F}$, Sholl profiles of basal dendrites of Layer V SMI-32-positive neurons in each hemisphere in two intact monkeys $(\boldsymbol{A}, \boldsymbol{B})$ and in three SCI monkeys $(\boldsymbol{D}-\boldsymbol{F})$. For each monkey, the IDCD are reported in the upper right angle. $\mathbf{G}$, Neuronal reconstruction example of apical dendrite of a Layer V SMI-32-positive neuron. $\boldsymbol{H}-\boldsymbol{L}$, Sholl profiles of apical dendrites of Layer V SMI-32-positive neurons in each hemisphere in two intact monkeys $(\boldsymbol{H}, \boldsymbol{I})$ and in three SCI monkeys $(\boldsymbol{J}-\boldsymbol{L})$. The curves represent the mean intersection values $\pm \mathrm{SD}$. As statistical test, a two-way ANOVA wit Bonferroni's multiple comparison post hoc test was performed $(* p \leq 0.05, * * p \leq 0.01$, $* * * p \leq 0.001)$. The $p$ values for the distance to soma main effect (up) and hemisphere main effect (right) are report for each monkey. \# in the upper right angle means a significative $p$ value for the interaction between the distance to soma and the hemisphere main effect.

Figure Contributions: A. Contestabile and M. Lucchini performed the microscopic analysis of the histologic sections. A. Contestabile analyzed the data. 
A

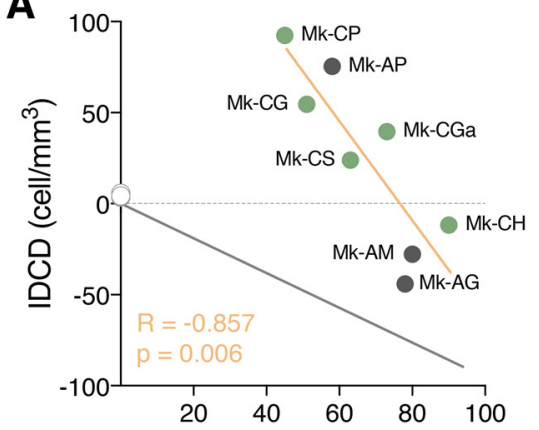

Percentage of hemisection extent (\%)

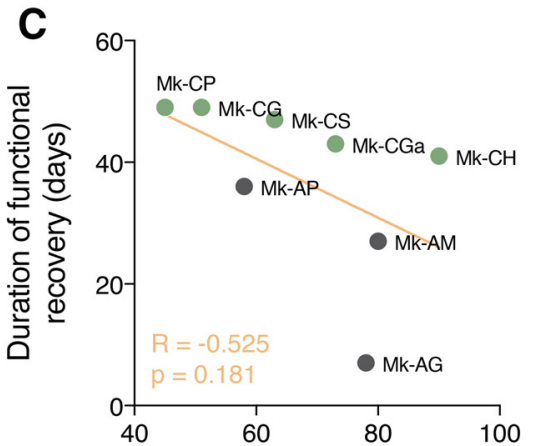

Percentage of hemisection extent (\%)
B

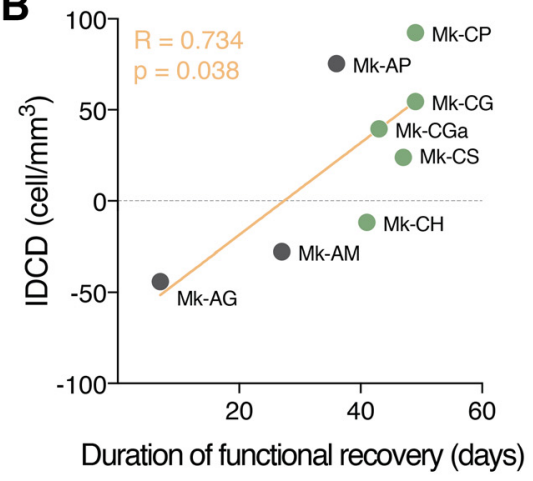

E

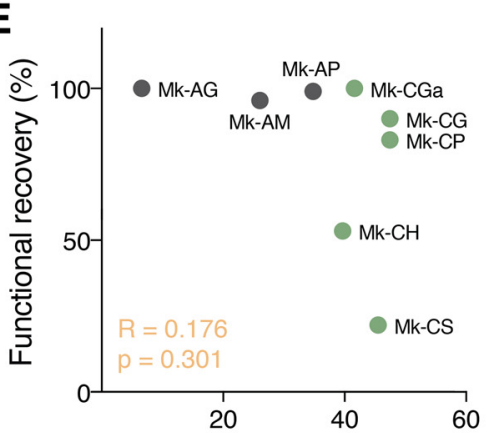

Duration of functional recovery (days)

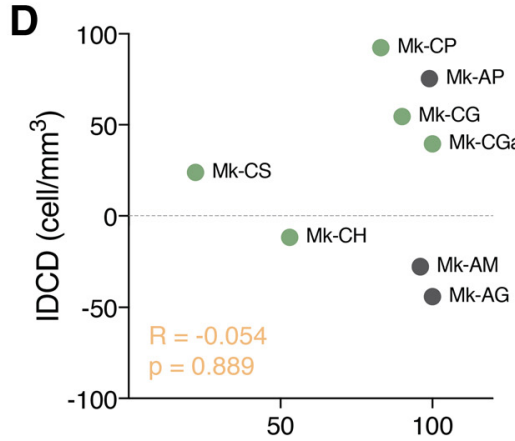

Functional recovery (\%)

$$
\begin{aligned}
& \text { Intact monkeys } \\
& \mathrm{SCl} \text { monkeys } \\
& \mathrm{SCl} \text { monkeys + Anti-Nogo A treatment } \\
& \text { Linear regression } \\
& \text { - Hypothesized interhemispheric } \\
& \text { cell density asymmetry }
\end{aligned}
$$

Figure 5. Relationship of IDCD, proportion of hemisection extent, duration of recovery and percentage of functional recovery. $\boldsymbol{A}$, The IDCD in F3 was plotted in function of the proportion of hemisection extent. The gray line represents the hypothesized interhemispheric cell density asymmetry (see Introduction). $\boldsymbol{B}$, The IDCD in F3 was plotted in function of the duration of functional recovery. $\boldsymbol{C}$, The duration of functional recovery was plotted in function of the proportion of hemisection extent. $\boldsymbol{D}$, The IDCD in F3 was plotted in function of the functional recovery. $\boldsymbol{E}$, The functional recovery of the animals was plotted in function of the duration of functional recovery. Intact (in white), SCl (light gray), and SCl-treated (dark gray) animals are divided by color code. In each graph, the $R$ and the $p$ value of the linear regression are reported.

Figure Contributions: A. Contestabile, R. Colangiulo, E. M. Rouiller, and E. Schmidlin performed the experiments and collected the data. A. Contestabile analyzed the data.

\section{Discussion}

The major conclusion of the present study is that a unilateral cervical cord hemisection retrogradely induced an interhemispheric asymmetry of SMI-32 staining in F3 Layer $\mathrm{V}$ in non-human primates, which is correlated with the extent of the $\mathrm{SCl}$ as well as the duration of functional recovery, but not with the extent (percentage) of the functional recovery. These conclusions are coherent with the observations described recently by our laboratory showing similar results in F3 Layer V in SMA after a unilateral M1 lesion (Contestabile et al., 2018). A coherent correlation between
IDCDs and lesion size as well as duration of functional recovery (but not extent of recovery) were observed in this study. In other words, the interhemispheric effects on SMI32 staining in F3 Layer V observed after M1 lesion (Contestabile et al., 2018) can be generalized to another type of unilateral lesion, namely a cervical cord hemisection. If the morphologic interhemispheric changes reflect variable balanced contributions of the two hemispheres in the process of functional recovery, then comparable mechanisms of functional recovery at cortical level may take place, regardless of the site of lesion 
(cortical or spinal), at least for injury restricted to one side of the brain or of the spinal cord.

The lateralization of the unilateral lesion, either in M1 or at cervical cord level, is of importance. The computation formula to establish the IDCD was chosen to be reversed in the present study (cervical cord lesion) as compared with our recent report on unilateral M1 lesion (Contestabile et al., 2018). The reason for such reversal is that the unilateral $\mathrm{M} 1$ lesion was on the same hemisphere as the F3 area, which exhibits (before the lesion) the quantitatively predominant corticocortical connection with the injured M1, whereas the transcortical connection from the opposite F3 is less strong (Contestabile et al., 2018, their Fig. 1). In case of cervical cord hemisection, the predominant CS projection from F3 is crossed (originating mainly from the opposite hemisphere), reason why the IDCD formula was reversed (see Materials and Methods). Because of this reversal, then the correlation plots in the M1 lesion model (Contestabile et al., 2018; their Fig. 5) and in the present study (Fig. 5) should appear similar, if the same correlations are verified. This is indeed the case for the correlation between IDCDs and the lesion extent (compare Contestabile et al., 2018, their Fig. 5B with the present Fig. $5 A$ ). In contrast, although there is a correlation in both lesion models between IDCDs and duration of functional recovery, the correlation was inverse in the M1 lesion model (Contestabile et al., 2018; their Fig. 5F) and positive in the present $\mathrm{SCl}$ model (Fig. $5 B$ ). As argued above, because of the reversal of the IDCD formula, we would have intuitively expected a similar correlation direction. This is not the case, suggesting that the mechanisms of recovery reflected by the correlation between IDCD and duration of functional recovery may be independent of the lateralization of the unilateral lesion, either cortical or cervical.

\section{Connectional diaschisis}

The results obtained in Contestabile et al. (2018), and in this study could be linked with the old concept of diaschisis (von Monakow, 1914), defined as a "loss of function and electrical activity in an area of the brain because of a lesion in a remote area that is neuronally connected with it," although it corresponds more to a "connectional diaschisis" (Carrera and Tononi, 2014; see Introduction). The unilateral SCI may have affected the morphology, phenotype and cellular expression of Layer $\mathrm{V}$ neurons in the contralesional hemisphere in a different manner with respect to the neurons localized in the ipsilesional hemisphere. This interhemispheric difference in phenotype could have influenced the affinity for the SMI-32 antibody and explain the observed interhemispheric asymmetry of density of SMI-32-positive Layer V neurons. This interpretation is moreover supported by the neuronal reconstruction. In fact, the directions of interhemispheric differences in dendritic arborization complexity (Fig. 4) were consistent with the direction of the IDCD, especially in the arborization of the basal dendrites, which are more implicated in the integration of the neuronal response and have a strong effect on action potential output because of their direct attachment to the cell body and the proximity to the axon (Nevian et al., 2007; Zhou et al., 2008). The changes of dendritic arborization following $\mathrm{SCl}$ can be linked to the recently published observation depicting that axotomy of peripheral motor projections induce changes in the dendritic arborization of M1 pyramidal neurons in the rodent model submitted to a permanent lesion of the facial nerve (Urrego et al., 2015). In another recent study aiming at measuring the structural changes in the CNS of human patients suffering from degenerative pathology affecting interaction between cortical motoneurons and spinal motoneurons such as amyotrophic lateral sclerosis (ALS) or dementia, the authors observed a degeneration of the apical dendrites of pyramidal neurons located in Layer $\mathrm{V}$ of M1 (Betz's cells as described in the paper), to a larger extent in patients suffering from ALS (Genç et al., 2017). These results are consistent with a lower dendritic arborization observed in the contralesional SMA (Fig. $4 G, L$ ) in animals having a larger extent of $\mathrm{SCl}$, as ALS is a major degenerative disease affecting a majority of motoneurons in the ventral horn of the spinal cord.

Interestingly, it seems that anti-Nogo-A antibody treatment does not have an effect on the observed interhemispheric difference in phenotype. The three treated animals showed results that are comparable to only lesioned animals. Neutralization of Nogo-A was found to promote sprouting of CS axons in macaque monkeys and to lead to a complete recovery of the manual dexterity after a unilateral hemisection lesion irrespectively on the lesion extent (Freund et al., 2006, 2007, 2009). However, it is possible that anti-Nogo-A antibody treatment could not preserve the phenotype of F3 Layer V pyramidal neurons since the SCl immediately affect the neuronal connection and altered the phenotype of the CS neurons in an irreversible manner.

\section{A possible role of the ipsilesional hemisphere during the functional recovery from $\mathrm{SCl}$}

The correlation between IDCD asymmetry and the duration of functional recovery is also reminiscent of the controversy related to the respective contributions of the ipsilesional versus contralesional hemispheres in the functional recovery following a unilateral $\mathrm{SCl}$. Previous studies in rodents (Ghosh et al., 2009) and humans (Lundell et al., 2011) demonstrated that the ipsilesional hemisphere underwent a profound reorganization of the motor areas after cervical SCl. Moreover, these previous findings suggest a correlation between brain activity, duration and extent of functional recovery and are consistent with our results. In fact, they revealed that monkeys with larger lesion tend to show shorter duration of functional recovery and negative IDCD suggesting a more prominent reorganization and a predominant contribution of the ipsilesional hemisphere (as compared with the contralesional one), immediately after severe SCl. On the other hand, the data also show that a more moderate lesion leads to a higher SMI-32-positive neurons density in the contralesional hemisphere suggesting a more important reorganization of the hemisphere predominantly affected by the spinal hemisection. Interestingly, previous studies reported an upregulation of the expression of Sema3A and 
NRP-1 in motoneurons located in the contralesional hemisphere after SCl in rats (De Winter et al., 2002; Hashimoto et al., 2004). It is likely that neurons whose descending fibers were transected upregulated the production of proteins involved in the regulation of axonal re-growth and maintenance. This phenomenon may possibly be correlated with the higher density of neurons in the contralesional hemisphere that express SMI-32, a major component of the neuronal cytoskeleton providing structural support to the axon. However, in a recent study based on meta-analysis of fMRI data assessing the possible reorganization of different regions of the cerebral cortex after $\mathrm{SCl}$ in human patients, the authors showed changes in bilateral SMA, but were unable to specify the type of change or potential role in the functional recovery (Wang et al., 2019).

\section{Limitations}

The present study involves a limited number of monkeys subjected to $\mathrm{SCl}(n=8)$, five of them received a control antibody, while three monkeys were subjected to anti-Nogo-A antibody treatment, as one may reasonably expect from a non-human primate study, mostly for ethical reasons. However, despite the limited number of monkeys, the data are internally coherent (e.g., F3 vs F6) and statistically significant correlations of IDCDs with lesion extent and duration of functional recovery emerged (Fig. 5). Furthermore, these correlation data are fully consistent with previous data derived from another pool of monkeys $(n=9)$ subjected to another type of unilateral lesion (M1) affecting the CS projection (Contestabile et al., 2018).

An important limitation of this study is because of the impossibility to compare the absolute values of SMI-32positive neurons' densities across animals. Although the raw data seems to show a higher density of SMI-32 neurons in both hemispheres of SCl monkeys as compared with intact animals, such interindividual comparison between the two groups of monkeys is problematic because of a very large interindividual variability of the histologic staining quality, strongly affecting the absolute quantification of neuronal density. The quality of SMI-32 staining in three of the four intact monkeys may have been different (lower) than in the SCI monkeys, leading to the differences in the absolute numbers of SMI-32-positive neurons between the two groups. In one of the intact monkeys (MkIC), the absolute cellular density is comparable to that of five of the $\mathrm{SCl}$ monkeys, corresponding thus to a partial overlap between the two groups (which are not statistically different with respect to their IDCDs in F3 and F6 (Fig. $3 B, D$ ). On the other hand, the quality of staining does not interfere when the comparison is intraindividual and furthermore restricted to a single histologic section (left hemisphere compared with the corresponding right hemisphere on the same section, both hemispheres processed histologically in the very same way and at the same time point).

Further limitations were related to the impossibility in some monkeys to perform the cell counting and consecutive IDCD analysis in F6 because of the lack of brain tissue. Finally, the histologic condition of some SMI-32 sections was not optimal to perform the neuronal dendritic reconstruction and for this reason the morphologic Sholl analysis was limited to five animals (Fig. 4).

\section{Conclusion}

Altogether, the current study on SCl and the recent investigation on M1 lesion (Contestabile et al., 2018) both show that a unilateral lesion affecting the CS projection system, either at its origin (in M1) or along its trajectory (at cervical level) results in a variable and adaptable interhemispheric balance of F3 Layer V pyramidal neurons, detected with the marker SMI-32, in a manner which is systematically correlated with the extent of the lesion as well as the duration of the functional recovery of manual dexterity. These observations suggest that the controversy on which hemisphere (ipsilesional vs contralesional) is involved in the functional recovery may be resolved by considering a contribution of both, however in an adaptable and finely tuned balance depending on the lesion properties (e.g., extent) and evolving with time during the recovery, the duration of the latter being a crucial factor in this process.

\section{References}

Beaud ML, Schmidlin E, Wannier T, Freund P, Bloch J, Mir A, Schwab ME, Rouiller EM (2008) Anti-Nogo-A antibody treatment does not prevent cell body shrinkage in the motor cortex in adult monkeys subjected to unilateral cervical cord lesion. BMC Neurosci 9:5.

Beaud ML, Rouiller EM, Bloch J, Mir A, Schwab ME, Wannier T, Schmidlin E (2012) Invasion of lesion territory by regenerating fibers after spinal cord injury in adult macaque monkeys. Neuroscience 227:271-282.

Boudrias MH, Lee SP, Svojanovsky S, Cheney PD (2010) Forelimb muscle representations and output properties of motor areas in the mesial wall of rhesus macaques. Cereb Cortex 20:704-719.

Carrera E, Tononi G (2014) Diaschisis: past, present, future. Brain 137:2408-2422.

Chao ZC, Sawada M, Isa T, Nishimura Y (2019) Dynamic reorganization of motor networks during recovery from partial spinal cord injury in monkeys. Cereb Cortex 29:3059-3073.

Contestabile A, Colangiulo R, Lucchini M, Gindrat A-D, Hamadjida A, Kaeser M, Savidan J, Wyss AF, Rouiller EM, Schmidlin E (2018) Asymmetric and distant effects of a unilateral lesion of the primary motor cortex on the bilateral supplementary motor areas in adult macaque monkeys. J Neurosci 38:10644-10656.

Courtine G, De Nunzio AM, Schmid M, Beretta MV, Schieppati M (2007) Stance- and locomotion-dependent processing of vibration-induced proprioceptive inflow from multiple muscles in humans. J Neurophysiol 97:772-779.

Dancause N, Barbay S, Frost SB, Plautz EJ, Chen D, Zoubina EV, Stowe AM, Nudo RJ (2005) Extensive cortical rewiring after brain injury. J Neurosci 25:10167-10179.

De Winter F, Oudega M, Lankhorst AJ, Hamers FP, Blits B, Ruitenberg MJ, Pasterkamp RJ, Gispen WH, Verhaagen J (2002) Injury-induced class 3 semaphorin expression in the rat spinal cord. Exp Neurol 175:61-75.

Dum RP, Strick PL (1996) Spinal cord terminations of the medial wall motor areas in macaque monkeys. J Neurosci 16:6513-6525.

Finger S, Koehler PJ, Jagella C (2004) The Monakow concept of diaschisis: origins and perspectives. Arch Neurol 61:283.

Fregosi M, Contestabile A, Hamadjida A, Rouiller EM (2017) Corticobulbar projections from distinct motor cortical areas to the reticular formation in macaque monkeys. Eur J Neurosci 45:13791395. 
Fregosi M, Contestabile A, Badoud S, Borgognon S, Cottet J, Brunet J-F, Bloch J, Schwab ME, Rouiller EM (2018) Changes of motor corticobulbar projections following different lesion types affecting the central nervous system in adult macaque monkeys. Eur $\mathrm{J}$ Neurosci 48:2050-2070.

Fregosi M, Rouiller EM (2017) Ipsilateral corticotectal projections from the primary, premotor and supplementary motor cortical areas in adult macaque monkeys: a quantitative anterograde tracing study. Eur J Neurosci 46:2406-2415.

Freund P, Schmidlin E, Wannier T, Bloch J, Mir A, Schwab ME, Rouiller EM (2006) Nogo-A-specific antibody treatment enhances sprouting and functional recovery after cervical lesion in adult primates. Nat Med 12:1220-1220.

Freund P, Wannier T, Schmidlin E, Bloch J, Mir A, Schwab ME, Rouiller EM (2007) Anti-Nogo-A antibody treatment enhances sprouting of corticospinal axons rostral to a unilateral cervical spinal cord lesion in adult macaque monkey. J Comp Neurol 502:644-659.

Freund P, Schmidlin E, Wannier T, Bloch J, Mir A, Schwab ME, Rouiller EM (2009) Anti-Nogo-A antibody treatment promotes recovery of manual dexterity after unilateral cervical lesion in adult primates - re-examination and extension of behavioral data. Eur $\mathrm{J}$ Neurosci 29:983-996.

Galea MP, Darian-Smith I (1997) Manual dexterity and corticospinal connectivity following unilateral section of the cervical spinal cord in the macaque monkey. J Comp Neurol 381:307-319.

Genç B, Jara JH, Lagrimas AKB, Pytel P, Roos RP, Mesulam MM, Geula C, Bigio EH, Özdinler PH (2017) Apical dendrite degeneration, a novel cellular pathology for Betz cells in ALS. Sci Rep 7:41765.

Geyer S, Zilles K, Luppino G, Matelli M (2000) Neurofilament protein distribution in the macaque monkey dorsolateral premotor cortex. Eur J Neurosci 12:1554-1566.

Ghosh A, Sydekum E, Haiss F, Peduzzi S, Zorner B, Schneider R, Baltes C, Rudin M, Weber B, Schwab ME (2009) Functional and anatomical reorganization of the sensory-motor cortex after incomplete spinal cord injury in adult rats. J Neurosci 29:1221012219.

Hashimoto M, Ino H, Koda M, Murakami M, Yoshinaga K, Yamazaki M, Moriya H (2004) Regulation of semaphorin 3A expression in neurons of the rat spinal cord and cerebral cortex after transection injury. Acta Neuropathol 107:250-256.

Hoogewoud F, Hamadjida A, Wyss AF, Mir A, Schwab ME, BelhajSaif A, Rouiller EM (2013) Comparison of functional recovery of manual dexterity after unilateral spinal cord lesion or motor cortex lesion in adult macaque monkeys. Front Neurol 4:101.

Isa $T$, Nishimura $Y$ (2014) Plasticity for recovery after partial spinal cord injury - hierarchical organization. Neurosci Res 78:3-8.

Jenny AB, Inukai J (1983) Principles of motor organization of the monkey cervical spinal cord. J Neurosci 3:567-575.

Kaeser M, Brunet JF, Wyss A, Belhaj-Saif A, Liu Y, Hamadjida A, Rouiller EM, Bloch J (2011) Autologous adult cortical cell transplantation enhances functional recovery following unilateral lesion of motor cortex in primates: a pilot study. Neurosurgery 68:14051416.

Kaeser M, Chatagny P, Gindrat AD, Savidan J, Badoud S, Fregosi M, Moret V, Roulin C, Schmidlin E, Rouiller E (2014) Variability of manual dexterity performance in non-human primates (Macaca fascicularis). Int J Comp Psychol 27:295-325.

Lawrence DG, Kuypers HGJM (1968) The functional organization of the motor system in the monkey: I. The effects of bilateral pyramidal lesions. Brain 91:15-36.

Lemon RN (2008) Descending pathways in motor control. Annu Rev Neurosci 31:195-218.

Liu J, Morel A, Wannier T, Rouiller EM (2002) Origins of callosal projections to the supplementary motor area (SMA): a direct comparison between pre-SMA and SMA-proper in macaque monkeys. J Comp Neurol 443:71-85.
Liu Y, Rouiller EM (1999) Mechanisms of recovery of dexterity following unilateral lesion of the sensorimotor cortex in adult monkeys. Exp Brain Res 128:149-159.

Lundell $H$, Christensen MS, Barthélemy $D$, Willerslev-Olsen $M$, Biering-Sørensen F, Nielsen JB (2011) Cerebral activation is correlated to regional atrophy of the spinal cord and functional motor disability in spinal cord injured individuals. Neuroimage 54:12541261.

Luppino G, Matelli M, Camarda R, Rizzolatti G (1994) Corticospinal projections from mesial frontal and cingulate areas in the monkey. Neuroreport 5:2545-2548.

Maier MA, Armand J, Kirkwood PA, Yang HW, Davis JN, Lemon RN (2002) Differences in the corticospinal projection from primary motor cortex and supplementary motor area to macaque upper limb motoneurons: an anatomical and electrophysiological study. Cereb Cortex 12:281-296.

McNeal DW, Darling WG, Ge J, Stilwell-Morecraft KS, Solon KM, Hynes SM, Pizzimenti MA, Rotella DL, Vanadurongvan T, Morecraft RJ (2010) Selective long-term reorganization of the corticospinal projection from the supplementary motor cortex following recovery from lateral motor cortex injury. J Comp Neurol 518:586-621.

Morecraft RJ, Ge J, Stilwell-Morecraft KS, McNeal DW, Hynes SM, Pizzimenti MA, Rotella DL, Darling WG (2015) Vulnerability of the medial frontal corticospinal projection accompanies combined lateral frontal and parietal cortex injury in rhesus monkey. J Comp Neurol 523:669-697.

Morecraft RJ, Ge J, Stilwell-Morecraft KS, McNeal DW, Hynes SM, Pizzimenti MA, Rotella DL, Darling WG (2016) Frontal and frontoparietal injury differentially affect the ipsilateral corticospinal projection from the nonlesioned hemisphere in monkey (Macaca mulatta). J Comp Neurol 524:380-407.

Nevian T, Larkum ME, Polsky A, Schiller J (2007) Properties of basal dendrites of layer 5 pyramidal neurons: a direct patch-clamp recording study. Nat Neurosci 10:206-214.

Nishimura Y, Isa T (2009) Compensatory changes at the cerebral cortical level after spinal cord injury. Neuroscientist 15:436-444.

Nishimura Y, Isa T (2012) Cortical and subcortical compensatory mechanisms after spinal cord injury in monkeys. Exp Neurol 235:152-161.

Nishimura $\mathrm{Y}$, Onoe $\mathrm{H}$, Morichika $\mathrm{Y}$, Perfiliev S, Tsukada $\mathrm{H}$, Isa $T$ (2007) Time-dependent central compensatory mechanisms of finger dexterity after spinal cord injury. Science 318:1150-1155.

Orczykowski ME, Arndt KR, Palitz LE, Kramer BC, Pessina MA, Oblak AL, Finklestein SP, Mortazavi F, Rosene DL, Moore TL (2018) Cell based therapy enhances activation of ventral premotor cortex to improve recovery following primary motor cortex injury. Exp Neurol 305:13-25.

Rathelot JA, Strick PL (2009) Subdivisions of primary motor cortex based on cortico-motoneuronal cells. Proc Natl Acad Sci USA 106:918-923.

Rouiller EM, Babalian A, Kazennikov O, Moret V, Yu XH, Wiesendanger M (1994) Transcallosal connections of the distal forelimb representations of the primary and supplementary motor cortical areas in macaque monkeys. Exp Brain Res 102:227-243.

Rouiller EM, Moret V, Tanné J, Boussaoud D (1996) Evidence for direct connections between the hand region of the supplementary motor area and cervical motoneurons in the macaque monkey. Eur J Neurosci 8:1055-1059.

Savidan J, Kaeser M, Belhaj-Saif A, Schmidlin E, Rouiller EM (2017) Role of primary motor cortex in the control of manual dexterity assessed via sequential bilateral lesion in the adult macaque monkey: a case study. Neuroscience 357:303-324.

Schmidlin E, Kaeser M, Gindrat AD, Savidan J, Chatagny P, Badoud S, Hamadjida A, Beaud ML, Wannier T, Belhaj-Saif A, Rouiller EM (2011) Behavioral assessment of manual dexterity in non-human primates. J Vis Exp. Advance online publication. Retrieved Nov 11, 2011. doi: $10.3791 / 3258$. 
Sternberger LA, Sternberger NH (1983) Monoclonal antibodies distinguish phosphorylated and nonphosphorylated forms of neurofilaments in situ. Proc Natl Acad Sci USA 80:6126-6130.

Sugiyama Y, Higo N, Yoshino-Saito K, Murata Y, Nishimura Y, Oishi $T$, Isa $T$ (2013) Effects of early versus late rehabilitative training on manual dexterity after corticospinal tract lesion in macaque monkeys. J Neurophysiol 109:2853-2865.

Urrego D, Troncoso J, Múnera A (2015) Layer 5 pyramidal neurons' dendritic remodeling and increased microglial density in primary motor cortex in a murine model of facial paralysis. Biomed Res Int 2015:1-11.

von Monakow C (1914) Die Lokalisation im Grosshirn und der Abbau der Funktion durch Kortikale Herde. JAMA 63:797.

Wang W, Xie W, Zhang Q, Liu L, Liu J, Zhou S, Shi J, Chen J, Ning B (2019) Reorganization of the brain in spinal cord injury: a meta-analysis of functional MRI studies. Neuroradiology 61:1309-1318.

Wannier T, Schmidlin E, Bloch J, Rouiller EM (2005) A unilateral section of the corticospinal tract at cervical level in primate does not lead to measurable cell loss in motor cortex. J Neurotrauma 22:703-717.

Yoshida Y, Isa T (2018) Neural and genetic basis of dexterous hand movements. Curr Opin Neurobiol 52:25-32.

Zaaimi B, Edgley SA, Soteropoulos DS, Baker SN (2012) Changes in descending motor pathway connectivity after corticospinal tract lesion in macaque monkey. Brain 135:2277-2289.

Zhou WL, Yan P, Wuskell JP, Loew LM, Antic SD (2008) Dynamics of action potential backpropagation in basal dendrites of prefrontal cortical pyramidal neurons. Eur J Neurosci 27:923-936. 